\title{
Spatial Dynamics of Tuned and Mistuned Bladed Disks with Cylindrical and Wedge-Shaped Friction Dampers
}

\author{
Lars Panning, Walter Sextro, and Karl Popp \\ Institute of Mechanics, University of Hannover, Hannover, Germany
}

One of the main tasks in the design of turbomachines like turbines, compressors, and fans is to increase the reliability and efficiency of the arrangement. Failures due to blade cracks are still a problem and have to be minimized with respect to costs and safety aspects. To reduce the maximum stresses, the blades can be coupled via friction damping devices such as underplatform dampers that are pressed onto the blade platforms by centrifugal forces. In this work, a method will be presented to optimize two different types of underplatform dampers in bladed disk applications with respect to a maximum damping effect.

In practice, underplatform dampers with various geometric properties-cylindrical and wedge-shaped-are commonly used and lead to different contact conditions. A discretization of the contact areas between the blade platforms and the dampers is applied to be able to investigate nearly arbitrary contact geometries and spatial blade vibrations. The functionality of the two mentioned damper types has been studied in detail under different working conditions of the assembly. The advantages and disadvantages of both damper types are pointed out and strategies are presented to improve the damper design. In this context, the influence of mistuning effects is discussed in terms of statistical mistuning of the blades' natural frequencies due to manufacturing tolerances as well as systematical mistuning due to a deliberate slight variation of the blade masses or geometries.

Keywords bladed disks, damper optimization, friction damping, mistuning

Received 25 June 2002; accepted 1 July 2002.

Address correspondence to Lars Panning, Institute of Mechanics, University of Hannover, Appelstrasse 11, D-30167 Hannover, Germany. E-mail: panning@ifm.uni-hannover.de
Friction damping devices such as underplatform dampers, tip shrouds, and damping wires are widely used in turbomachinery applications to reduce blade vibration amplitudes and resonance stresses to a reasonable amount. In the case of blade vibrations, relative displacements between the blades or between the friction damping device and the neighboring blades occur, and friction forces are generated that provide additional damping to the structure due to energy dissipation. Underplatform dampers are mounted between adjacent blades and pressed onto the blade platforms by centrifugal forces during operation of the assembly.

Various types of underplatform dampers have been investigated (cylindrical dampers: Csaba, 1999; Jareland, 2001b; Pfeiffer and Hajek, 1992; Sextro et al., 1997; wedge-shaped dampers: Jareland, 2001a; Sanliturk et al., 1999; Yang and Menq, 1997). A comparison of damper types can be found in Panning and colleagues (2000).

Periodic structures with mistuning effects provide a wide field of research, as the number of publications shows (Ewins, 1968; Judge and Pierre, 2000; Mignolet et al., 1998). Sextro and colleagues (1998) have investigated a bladed disk with cylindrical friction dampers and frequency mistuning; Chen and Sinha (1989) analyzed the distribution of the vibration amplitudes of a mistuned periodic structure, including friction; Cha and Sinha (2000) investigated a mistuned system with friction and nonharmonic excitation forces; Jareland and Csaba (2000) studied a bladed disk with friction dampers, including mistuned damper properties.

The objective of the present work is the derivation of a general model to analyze the effectiveness of various damper types applied to tuned as well as to mistuned systems. The model makes use of a spatial formulation of the blade dynamics and contact properties in order to analyze real bladed systems. A spatial contact model is presented that considers flat as well as curved contact surfaces with respect to the damper types depicted in Figure 1. Both damper types are compared with respect to the friction damping they provide to the structure under a variety 


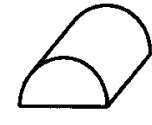

Cylindrical damper

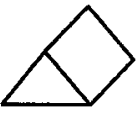

Wedge-shaped (cottage roof) damper

FIGURE 1

Typical underplatform damper types.

of working conditions, and strategies are derived to improve the damper design - for example, asymmetric blade platforms and the utilization of mistuning effects.

\section{MODELING OF BLADED DISKS WITH UNDERPLATFORM FRICTION DAMPERS}

\section{Modal Description of the Linear Structures}

The dynamic behavior of an individual blade of an assembly can be described after discretization by means of the ordinary differential equation

$$
\mathbf{M u ̈}(t)+\mathbf{D} \dot{\mathbf{u}}(t)+\mathbf{K u}(t)=\mathbf{p}_{\mathrm{E}}(t)+\mathbf{p}_{\mathrm{C}}(t)
$$

where the right-hand side of Equation (1) contains external excitation forces $\mathbf{p}_{\mathrm{E}}(t)$ and contact forces $\mathbf{p}_{\mathrm{C}}(t)$ acting between neighboring blades via the friction damper. $\mathbf{M}, \mathbf{D}$, and $\mathbf{K}$ denote the mass, damping, and stiffness matrix of the blade and $\mathbf{u}(t)$ indicates the vector of the generalized displacements. Following the assumption of small vibration amplitudes, gyroscopic effects are neglected. Nevertheless, centrifugal stiffening (CF) effects can be included in a speed-dependent stiffness matrix that can be provided by common finite element codes. Each blade is coupled with its neighbors by the internal contact forces $\mathbf{p}_{\mathrm{C}}(t)$ via the blade-damper interface. In order to reduce the number of DOF included in Equation (1), the modal transformation

$$
\mathbf{u}(t)=\mathbf{\Phi q}(t)
$$

is applied, making use of the modal matrix $\boldsymbol{\Phi}$ that contains $n_{\mathrm{m}}$ mass-normalized eigenvectors of the linear, undamped blade; $\mathbf{q}(t)$ denotes the vector of the modal amplitudes. The external excitation forces $\mathbf{p}_{\mathrm{E}}(t)$ are assumed to be harmonic and fixed in the inertial system. In practical applications, the excitation forces include the fundamental harmonic as well as a number of multiples of the angular rotational speed such as those due to the number of annular burners in a combustion chamber of a gas turbine or the number of preceding vanes. In many cases, one harmonic plays a dominant role in the excitation spectrum and, thus, only monofrequent excitation forces are considered. The angular excitation frequency $\Omega_{\mathrm{E}}$ is defined by the angular rotational speed of the assembly $\Omega_{\mathrm{R}}$ and the engine order $E O$ by

$$
\Omega_{\mathrm{E}}=E O \Omega_{\mathrm{R}}
$$

The time-variant contact forces are of a nonlinear nature due to dry friction and can show transitions between sticking and slipping conditions of the damper. A numerical time integration of the entire system with a large number of DOF will lead to an enormous computational effort, especially in the case of a mistuned system. In order to develop a fast algorithm with the objective of improving the damper design, the nonlinear periodic contact forces are developed in a Fourier series that is truncated after the fundamental harmonic, where the Fourier coefficients are a function of the amplitude of the relative displacements between the contacting bodies. This harmonic balance method (HBM) has been proven to be a powerful method of analyzing systems with dry friction accurately and more quickly than timestep integration methods and is applied here to an appropriate contact model. After linearizing the contact forces by means of the HBM and using the modal transformation given in Equation (2), Equation (1) may be rewritten in complex notation as

$$
\hat{\mathbf{S}} \hat{\mathbf{q}}=\boldsymbol{\Phi}^{\mathbf{T}}\left(\hat{\mathbf{p}}_{\mathrm{E}}+\hat{\mathbf{p}}_{\mathrm{C}}\right)
$$

where

$$
\hat{\mathbf{S}}=\operatorname{diag}\left(\omega_{0 j}^{2}-\Omega_{\mathrm{E}}^{2}+\mathrm{i} 2 D_{j} \omega_{0 j} \Omega_{\mathrm{E}}\right), \quad j=1(1) n_{\mathrm{m}}
$$

denotes the diagonalized dynamic stiffness matrix, containing the natural angular frequencies $\omega_{0 j}$ and the modal damping ratios $D_{j}$ of the $n_{\mathrm{m}}$ considered vibration modes of the blade; $\hat{\mathbf{q}}$ is the vector of modal amplitudes, $i$ is the imaginary unit, and $\left(^{\wedge}\right)$ indicates complex quantities.

\section{Contact Kinematics}

To determine the generalized contact forces, the generalized relative displacements between the damper and the blade platforms have to be known (Fig. 2). OL and OR denote the so-called control points in the center of each nominal contact area. $\mathrm{OL}^{\prime}$ and $\mathrm{OR}^{\prime}$ indicate the coinciding points at the damper. In the case of spatial vibrations, three translational and three rotational relative displacements have to be determined for the left and right contact points, respectively. These generalized relative displacements can be expressed as

$$
\hat{\mathbf{w}}=\mathbf{G}_{\mathrm{D}} \hat{\mathbf{u}}_{\mathrm{D}}-\mathbf{G}_{\mathrm{P}} \hat{\mathbf{u}}_{\mathrm{P}}
$$

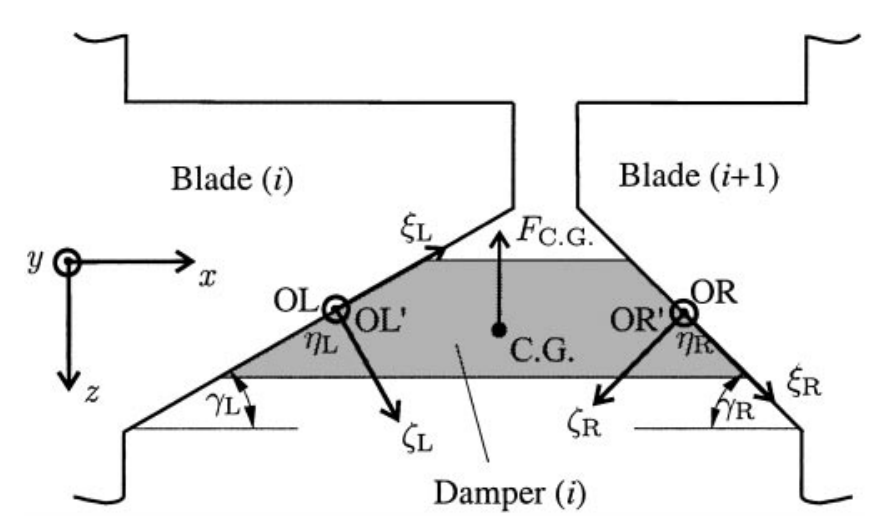

FIGURE 2

Contact geometry. 
where

$$
\hat{\mathbf{w}}=\left[\hat{w}_{u_{\xi}, \mathrm{OL}^{\prime}} \ldots \hat{w}_{\varphi_{\zeta}, \mathrm{OL}^{\prime}} \ldots \hat{w}_{u_{\xi}, \mathrm{OR}^{\prime}} \ldots \hat{w}_{\varphi_{\zeta}, \mathrm{OR}^{\prime}}\right]^{\mathbf{T}}
$$

The vector of the generalized platform displacements is expressed as

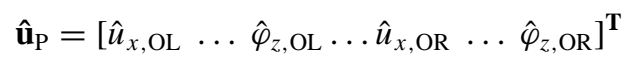

and the generalized rigid body displacements of the friction damper as

$$
\hat{\mathbf{u}}_{\mathrm{D}}=\left[\begin{array}{lll}
\hat{u}_{x, \mathrm{D}} & \ldots \hat{\varphi}_{z, \mathrm{D}}
\end{array}\right]^{\mathbf{T}}
$$

with respect to the displacement of the center of gravity $(\mathrm{CG})$ and the real-valued geometry matrixes $\mathbf{G}_{\mathrm{D}}$ and $\mathbf{G}_{\mathrm{P}}$, which contain the geometric properties of the damper and the adjacent platforms, e.g., the platform angles $\gamma_{\mathrm{L}}$ and $\gamma_{R}$ (see Fig. 2 and Panning et al., 2000).

\section{Contact Model}

The calculation of the contact forces in the case of spatial blade vibrations requires a contact model that considers contact forces as well as contact moments within the contact areas. A spatial contact model that is based on a discretization of the contact zones into several point contact elements has been proposed by Sextro (1999). The basic features of the contact model are as follows.

First, the regarded contact area of length $\ell_{\mathrm{C}}$ and height $h_{\mathrm{C}}$ is discretized into several point contact elements, as depicted in Figure 3. In the tangential direction, the point contact model is based on a Masing element that has been extended to twodimensional motions, consisting of a Coulomb friction element and a tangential point contact stiffness $\Delta c_{\mathrm{T}}$ in series. Additionally, a normal point contact stiffness $\Delta c_{\mathrm{N}}$ is included so as to

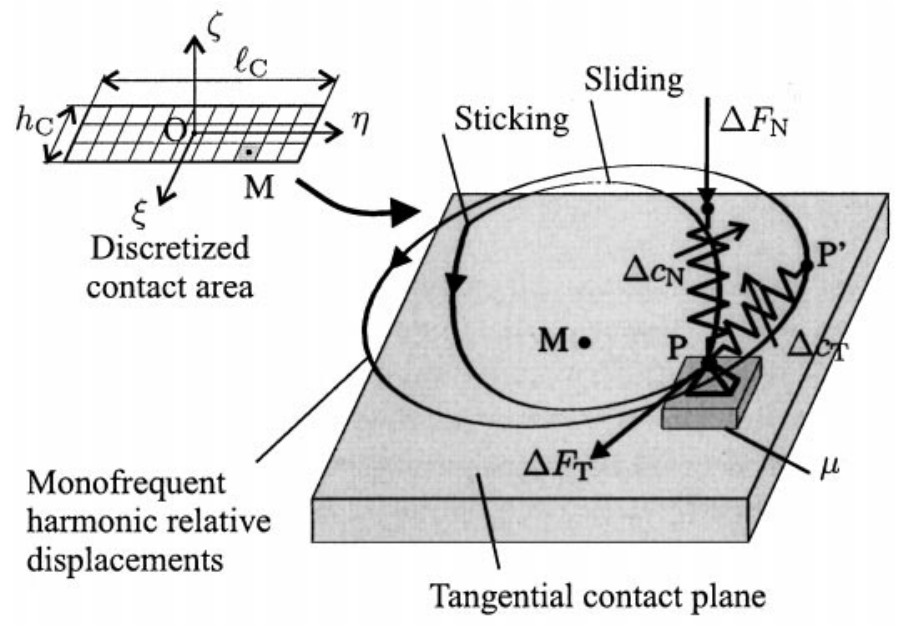

FIGURE 3

Three-dimensional point contact model. consider the elasticity of the blade platforms and the damper in the normal $(\zeta-)$ direction. The normal and tangential stiffnesses are nonlinearly dependent on the normal relative displacements due to the surface roughness of the contacting bodies that causes an increasing percentage contact area with higher normal forces. The relative displacements of an arbitrary point $\mathrm{P}^{\prime}$ of the contact area can be calculated by means of the relative displacements of the control point $\mathrm{OL}^{\prime}$ of the left and $\mathrm{OR}^{\prime}$ of the right contact area, respectively (see Equation (7)) and its position within the contact area). The point contact forces $\Delta F_{\mathrm{N}}$ and $\Delta F_{\mathrm{T}}$ of every single point contact are calculated by a nonlinear relationship between the relative displacements and the corresponding point contact stiffnesses. Assuming monofrequent harmonic relative displacements, the contact forces become nonlinear because of the nonlinear point contact stiffnesses and stick-slip transitions in the tangential contact plane. The nonlinear periodic point contact forces are linearized by means of the harmonic balance method (HBM), as mentioned above. Finally, all point contact forces are summarized with respect to the control point to give six generalized contact forces acting between the damper and the blade platforms within each contact area. From the contact forces $\hat{\mathbf{F}}_{\mathrm{C}}$ and the relative displacements $\hat{\mathbf{w}}$ from Equation (6), a nonlinear diagonalized contact stiffness matrix is generated:

$$
\hat{\mathbf{K}}_{\mathrm{C}}=\operatorname{diag}\left[\hat{k}_{u_{\xi}, \mathrm{OL}} \ldots \hat{k}_{\varphi_{\zeta}, \mathrm{OL}} \hat{k}_{u_{\xi}, \mathrm{OR}} \ldots \hat{k}_{\varphi_{\zeta}, \mathrm{OR}}\right]
$$

It yields

$\hat{\mathbf{F}}_{\mathrm{C}}=\left[\begin{array}{llllll}\hat{F}_{\mathrm{C} \xi, \mathrm{OL}} & \ldots & \hat{M}_{\mathrm{C} \zeta, \mathrm{OL}} & \hat{F}_{\mathrm{C} \xi, \mathrm{OR}} & \ldots & \hat{M}_{\mathrm{C} \zeta, \mathrm{OR}}\end{array}\right]^{\mathbf{T}}=\hat{\mathbf{K}}_{\mathrm{C}}(\hat{\mathbf{w}}) \hat{\mathbf{w}}$

where the generalized contact forces $\hat{\mathbf{F}}_{\mathrm{C}}$ acting on the blade platforms in OL and OR, respectively, are given in platform coordinates $\xi, \eta, \zeta . \hat{\mathbf{K}}_{\mathrm{C}}$ contains 12 complex contact stiffnesses, 6 for each contact area, depending on the amplitudes of the relative displacements and including stiffness (real part) as well as damping (imaginary part) properties. With increasing tangential displacement, the real part of the complex stiffness decreases because of the predominantly sliding condition of the contact points, indicating a softening stiffness characteristic. Slight jump phenomena in the frequency response function (FRF) of the system have been observed under certain parameter conditions.

\section{Damper Kinetics}

To determine the generalized relative displacements $\hat{\mathbf{w}}$ within the contact zones, the generalized rigid body displacements $\hat{\mathbf{u}}_{\mathrm{D}}$ of the friction damper, represented by the motion of its center of gravity, CG, are required; see Equation (6). They can be determined by the formulation of the equations of motion of the damper. Assuming monofrequent damper displacements, one obtains

$$
-\Omega_{\mathrm{E}}^{2} \mathbf{I}_{\mathrm{D}} \hat{\mathbf{u}}_{\mathrm{D}}=-\mathbf{G}_{\mathrm{D}}^{\mathrm{T}} \hat{\mathbf{F}}_{\mathrm{C}}
$$


with the generalized mass matrix $\mathbf{I}_{\mathrm{D}}$ of the damper and the generalized friction forces $\hat{\mathbf{F}}_{\mathrm{C}}$ acting on the damper at the left and right contacts. In many practical applications, the inertia properties of the damper may be neglected because of a small damper mass. Hence, the left-hand side of Equation (12) equals zero.

\section{Bladed Disk Model}

The contact forces $\hat{\mathbf{p}}_{\mathrm{C}}$ acting on the platforms of an individual blade can be found by combining the basic equations of the contact kinematics, the contact model, and the damper kinetics. By inserting Equations (6) and (11) into Equation (12), the damper displacements $\hat{\mathbf{u}}_{\mathrm{D}}$ can be determined. Inserting this expression for $\hat{\mathbf{u}}_{\mathrm{D}}$ into Equation (6) yields the nonlinear expression

$$
\hat{\mathbf{w}}=\hat{\mathbf{N}}(\hat{\mathbf{w}}) \mathbf{G}_{\mathrm{P}} \hat{\mathbf{u}}_{\mathrm{P}}
$$

with

$$
\hat{\mathbf{N}}(\hat{\mathbf{w}})=\mathbf{G}_{\mathrm{D}}\left[\mathbf{G}_{\mathrm{D}}^{\mathrm{T}} \hat{\mathbf{K}}_{\mathrm{C}}(\hat{\mathbf{w}}) \mathbf{G}_{\mathrm{D}}-\Omega_{\mathrm{E}}^{2} \mathbf{I}_{\mathrm{D}}\right]^{-1} \mathbf{G}_{\mathrm{D}}^{\mathrm{T}} \hat{\mathbf{K}}_{\mathrm{C}}(\hat{\mathbf{w}})-\mathbf{E}
$$

and $\mathbf{E}$ as unity matrix. Inserting Equation (13) into Equation (11) and transforming the contact forces $\hat{\mathbf{F}}_{\mathrm{C}}$ into blade coordinates $x, y, z$ yields the contact forces $\hat{\mathbf{F}}_{\mathrm{P}}$ acting on the platforms of the adjacent blades of each damper;

$$
\hat{\mathbf{F}}_{\mathrm{P}}=\mathbf{G}_{\mathrm{P}}^{\mathrm{T}} \hat{\mathbf{K}}_{\mathrm{C}}(\hat{\mathbf{w}}) \hat{\mathbf{N}}(\hat{\mathbf{w}}) \mathbf{G}_{\mathrm{P}} \hat{\mathbf{u}}_{\mathrm{P}}
$$

The vector of the modal contact forces ${ }^{(i)} \boldsymbol{\Phi}_{\mathrm{P}}^{\mathrm{T}(i)} \hat{\mathbf{p}}_{\mathrm{C}}$ acting on each blade $(i)$ consists of the contact forces in the point OL between the $i$ th blade and the $i$ th friction damper and the contact forces in OR between the $i$ th blade and the damper $(i-1)$. Expressing the platform displacements ${ }^{(i)} \hat{\mathbf{u}}_{\mathrm{P}}$ of the $i$ th blade by means of the modal amplitudes ${ }^{(i)} \hat{\mathbf{q}}$ and the modal matrixes ${ }^{(i)} \boldsymbol{\Phi}_{\mathrm{OL}}$ and ${ }^{(i)} \boldsymbol{\Phi}_{\mathrm{OR}}$ leads to the system equation of an $n$-bladed disk assembly with $n$ friction dampers:

$$
\left[\hat{\mathbf{S}}_{\mathrm{As}}-\boldsymbol{\Phi}_{\mathrm{P}, \mathrm{As}}^{\mathrm{T}} \hat{\mathbf{K}}_{\mathrm{C}, \mathrm{As}} \boldsymbol{\Phi}_{\mathrm{P}, \mathrm{As}}\right] \hat{\mathbf{q}}_{\mathrm{As}}=\boldsymbol{\Phi}_{\mathrm{E}, \mathrm{As}}^{\mathrm{T}} \hat{\mathbf{p}}_{\mathrm{E}, \mathrm{As}}
$$

with

$$
\begin{aligned}
\hat{\mathbf{S}}_{\mathrm{As}} & =\operatorname{diag}\left({ }^{(i)} \hat{\mathbf{S}}\right) \\
\hat{\mathbf{K}}_{\mathrm{C}, \mathrm{As}} & =\operatorname{diag}\left({ }^{(i)} \mathbf{G}_{\mathrm{P}}^{\mathrm{T}}{ }^{(i)} \hat{\mathbf{K}}_{\mathrm{C}}\left({ }^{(i)} \hat{\mathbf{w}}\right)^{(i)} \hat{\mathbf{N}}\left({ }^{(i)} \hat{\mathbf{w}}\right){ }^{(i)} \mathbf{G}_{\mathrm{P}}\right) \\
\hat{\mathbf{q}}_{\mathrm{As}} & =\left[{ }^{(1)} \hat{\mathbf{q}}^{\mathrm{T}}{ }^{\left({ }^{(2)}\right.} \hat{\mathbf{q}}^{\mathrm{T}} \ldots{ }^{(n)} \hat{\mathbf{q}}^{\mathrm{T}}\right]^{\mathrm{T}}
\end{aligned}
$$

and

$\boldsymbol{\Phi}_{\mathrm{P}, \mathrm{As}}=\left[\begin{array}{cccc}{ }^{(1)} \boldsymbol{\Phi}_{\mathrm{OL}} & \mathbf{0} & \cdots & \mathbf{0} \\ \mathbf{0} & { }^{(2)} \boldsymbol{\Phi}_{\mathrm{OR}} & & \vdots \\ & { }^{(2)} \boldsymbol{\Phi}_{\mathrm{OL}} & & \\ \vdots & & \ddots & \mathbf{0} \\ & & & { }^{(n)} \boldsymbol{\Phi}_{\mathrm{OR}} \\ \mathbf{0} & & & { }^{(n)} \boldsymbol{\Phi}_{\mathrm{OL}} \\ { }^{(1)} \boldsymbol{\Phi}_{\mathrm{OR}} & \mathbf{0} & \cdots & \mathbf{0}\end{array}\right], \quad i=1(1) n . \quad[20]$

Mistuning effects can be considered with regard to the dynamic properties of each individual blade (natural frequencies, modal damping ratios, and mode shapes) as well as the contact properties (damper mass, coefficient of friction, and contact geometry). In the case of a perfectly tuned system, cyclic boundary conditions may be used to reduce the computational effort remarkably. Equation (16) then can be simplified to one representative blade:

$$
\begin{aligned}
\hat{\mathbf{S}}_{\mathrm{As}} & =\hat{\mathbf{S}} \\
\hat{\mathbf{K}}_{\mathrm{C}, \mathrm{As}} & =\mathbf{G}_{\mathrm{P}}^{\mathrm{T}} \hat{\mathbf{K}}_{\mathrm{C}}(\hat{\mathbf{w}}) \hat{\mathbf{N}}(\hat{\mathbf{w}}) \mathbf{G}_{\mathrm{P}} \\
\hat{\mathbf{q}}_{\mathrm{As}} & =\hat{\mathbf{q}} \\
\hat{\boldsymbol{\Phi}}_{\mathrm{P}, \mathrm{As}} & =\left[\begin{array}{c}
\boldsymbol{\Phi}_{\mathrm{OL}} \\
\mathrm{e}^{-i \Delta \varphi} \boldsymbol{\Phi}_{\mathrm{OR}}
\end{array}\right]
\end{aligned}
$$

The modal matrix $\hat{\boldsymbol{\Phi}}_{\mathrm{P}, \mathrm{As}}$ becomes complex-valued because of the interblade phase angle:

$$
\Delta \varphi=\frac{2 \pi E O}{n}
$$

\section{TUNED SYSTEM}

\section{Spatial Blade Vibrations}

Neglecting manufacturing tolerances and assuming a perfectly tuned bladed disk in the first step, the calculation of the forced response of the entire assembly can be reduced to a single blade using Equations (21) through (25). In the following study, a tuned bladed disk is investigated; it consists of $n=16$ identical, clamped, simplified model blades, as depicted in Figure 4, and an engine order $E O=1$. The platform angles are $\gamma_{\mathrm{L}}=\gamma_{\mathrm{R}}=35^{\circ}$. The spatial dynamics of the symmetrical beamlike blade with a constant rectangular cross-section are considered by the first four vibration modes, including two flatwise bending $(1 \mathrm{~F}, 2 \mathrm{~F})$, one edgewise bending (1E), and one torsional mode (1T). The natural frequencies and modal damping ratios used during the simulations are given in Table 1.

Figure 4 shows the FRF in the range of the first four modes introducing cylindrical dampers with a damper mass of $m=1 \mathrm{~g}$. The excitation forces are set to $\hat{F}_{x}=0.1 \mathrm{~N} ; \hat{F}_{y}=-0.1 \mathrm{~N}$; and $\hat{M}_{z}=0.001 \mathrm{Nm}$, leading to spatial blade vibrations. Comparing the results with those of the assembly without friction dampers, 


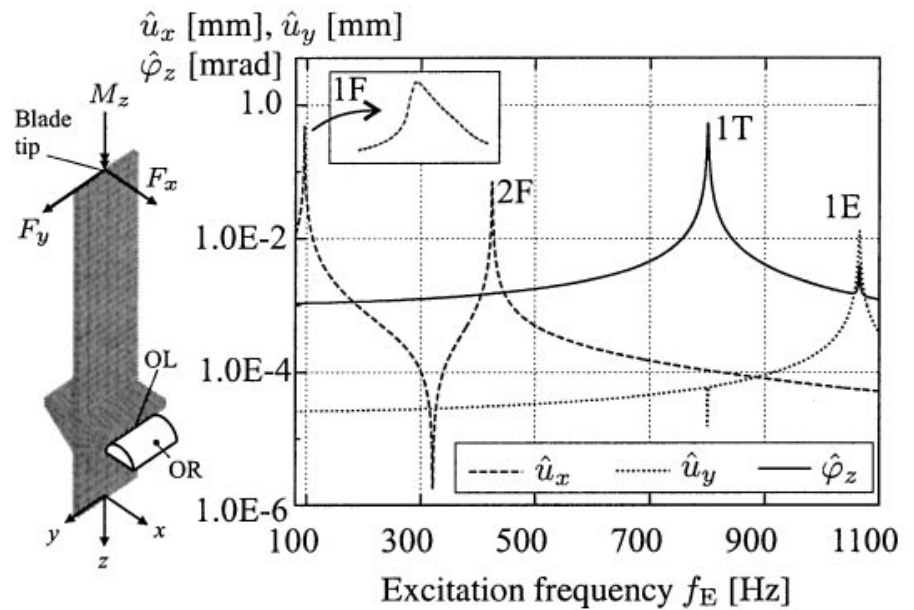

FIGURE 4

Blade model and FRF in the range of the first four vibration modes, including a well-damped first bending mode (1F) with a cylindrical damper.

two main results should be mentioned. The first flatwise vibration mode is damped very well; the amplitude reduction is about $71 \%$ if an optimal damper mass is chosen. The resonance frequencies of the other modes experience only a slight shift to higher values due to the additional stiffness that is introduced into the system. The additional damping that is provided by the friction damper for these modes is negligible because the damper is optimized for the first mode, and the chosen damper mass is too high to lead to sufficient relative displacements in the case of the higher vibration modes. Here, the damper sticks because of higher centrifugal forces with increasing rotational speed and smaller relative displacements at higher modes. The slight amplitude reduction of these modes is caused by a higher resonance frequency rather than by friction damping. Furthermore, it becomes evident that the uncoupled modes of the symmetrical beamlike blade without a damper are coupled in the case with friction dampers because of the contact kinematics of the bladedamper-blade structure and the damper kinetics. In particular, the coupling of torsional (1T) and edgewise bending vibration (1E) becomes obvious. These effects may demonstrate the necessity and effectiveness of a spatial model to predict the forced blade vibrations with satisfying accuracy.

TABLE 1

Frequency shift and amplitude reduction using a cylindrical damper with $m=1 \mathrm{~g}$

\begin{tabular}{ccrcrc}
\hline$j$ & Mode & \multicolumn{1}{c}{$\begin{array}{c}f_{0 j} \\
(\mathrm{~Hz})\end{array}$} & $\begin{array}{c}D_{j} \\
(-)\end{array}$ & $\begin{array}{c}f_{\text {Res,damped }} \\
(\mathrm{Hz})\end{array}$ & $\begin{array}{c}\text { Amplitude } \\
\text { reduction }(\%)\end{array}$ \\
\hline 1 & $1 \mathrm{~F}$ & 95.0 & 0.001 & 95.3 & 71.1 \\
2 & $2 \mathrm{~F}$ & 423.0 & 0.001 & 424.4 & 1.5 \\
3 & $1 \mathrm{~T}$ & 783.0 & 0.001 & 799.5 & 5.0 \\
4 & $1 \mathrm{E}$ & 1065.0 & 0.001 & 1065.1 & 0.3 \\
\hline
\end{tabular}

For clarity, the following investigations are focused on the first flatwise vibration mode, and the excitation force was set to $\hat{F}_{x}=0.1 \mathrm{~N}$ for simplicity. Additional vibration modes and real turbine blades, including complex coupled spatial vibration modes and distributed excitation forces acting on the airfoil, could be analyzed easily without any changes in or adjustments to the developed model.

\section{Optimization of the Damper Mass}

In order to gain the maximum damping effect from a cylindrical damper, the damper mass $m$ is one of the most important parameters, and it can be easily varied to some extent in the real turbine blade assembly. Thus, the damper mass is optimized in the first step for the given sample configuration. Figure 5 shows the blade tip amplitude $\hat{u}_{x}$ in the range of the first bending mode for $E O=1$ and different damper masses from $m=0 \mathrm{~g}$, representing the blade vibrations without any damper and leading to a resonance amplitude of $\hat{u}_{x, \text { Res }}=1.7 \mathrm{~mm}$ and $m=1 \mathrm{~kg}$. The damper sticks due to very high contact normal forces and, thus, no friction damping can be provided. Inbetween those limits, the optimal damper mass can be found to be $m_{\mathrm{Opt}}=1 \mathrm{~g}$, leading to a resonance amplitude of about $\hat{u}_{x, \text { Res }}=0.5 \mathrm{~mm}$.

Comparing the effectiveness of a cylindrical and a wedgeshaped damper with a chosen contact height of $h_{\mathrm{C}}=2 \mathrm{~mm}$, an optimization of each damper was performed with regard to its mass. The damping potential of both damper types for the regarded engine order $E O=1$ is given in Figure 6, where it becomes obvious that the optimized wedge-shaped damper leads to lower resonance amplitudes and a smaller optimal damper mass. The reason for the better damping effect of the wedgeshaped damper becomes clearer if both damper types are studied for different engine orders.

In Figure 7, the resonance amplitude is plotted against the engine order, which varies from $E O=0$ (in phase vibration, umbrella mode) to $E O=8$ (antiphase vibration of neighboring blades) using a cylindrical and a wedge-shaped damper, each

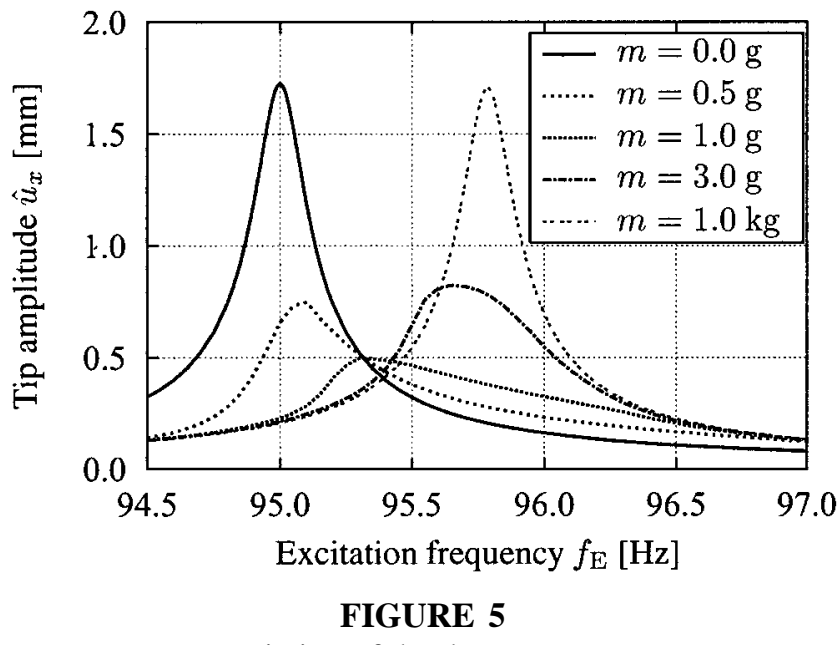

Variation of the damper mass. 


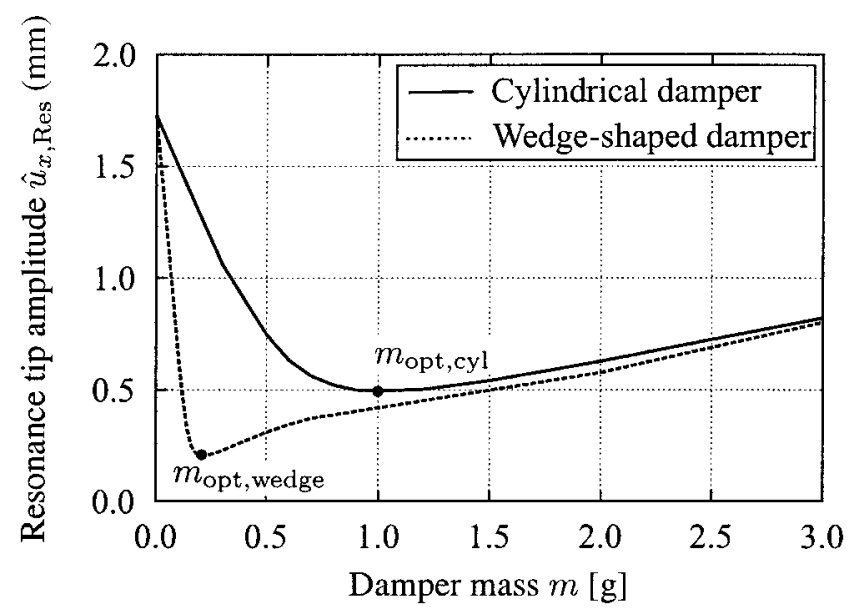

FIGURE 6

Optimal damper mass of both damper types for engine order 1 .

optimized with respect to its mass for the actual engine order. Figure 8 shows the corresponding optimal damper masses. In the case of engine order $E O=0$, all blades experience the same vibration amplitudes simultaneously. Because of the damper's geometry, the cylindrical damper gets in rolling condition and the damping effect vanishes due to a lack of relative motions in the contact areas. Hence, the vibration amplitudes equal those of the blades without any damper, and an optimization of the damper mass is not possible as a result of vanishing relative displacements. At higher engine orders, the possible amplitude reduction increases for both damper types due to larger relative displacements. The corresponding optimal damper mass increases for higher engine orders as well. To get resonance effects at given angular excitation frequency $\Omega_{\mathrm{E}}$, the corresponding rotation angular frequency $\Omega_{\mathrm{R}}$ is defined by Equation (3). Thus, at the same excitation angular frequency, the centrifugal forces

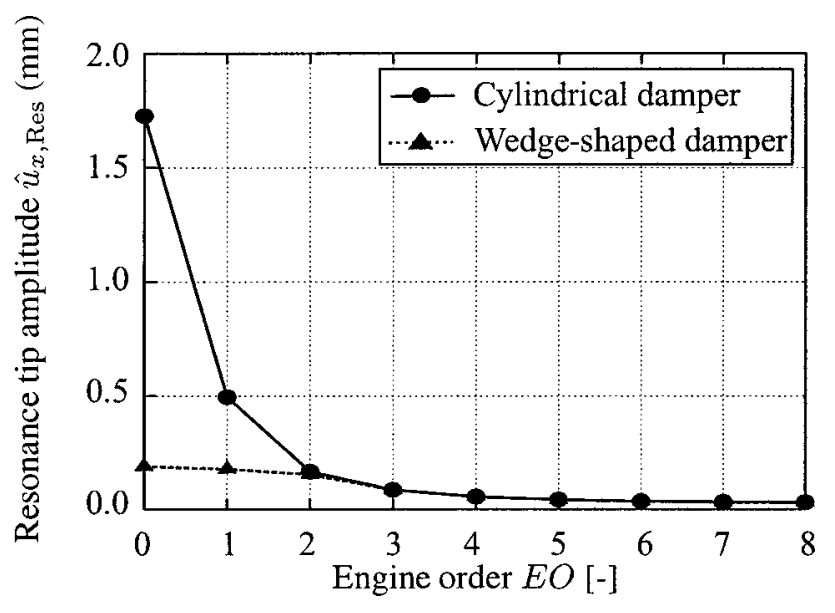

FIGURE 7

Achievable amplitude reduction at differing engine orders using a cylindrical or a wedge-shaped damper.

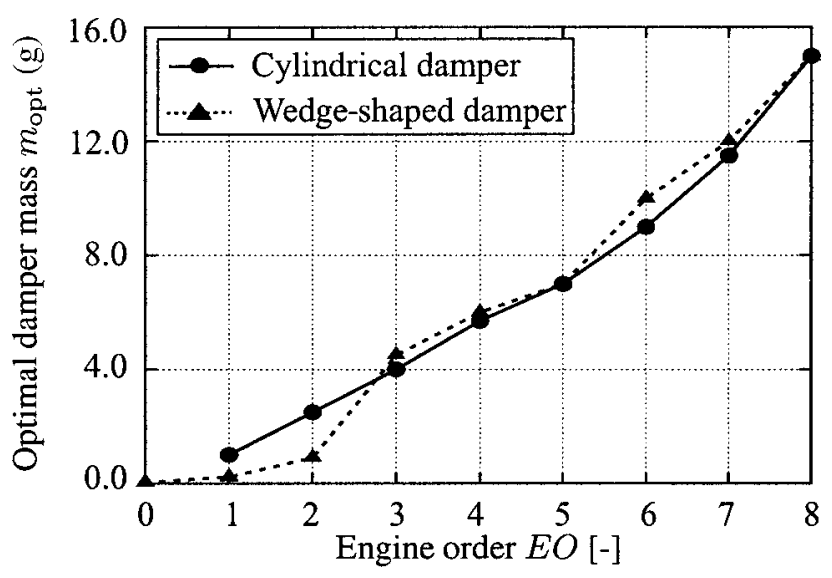

FIGURE 8

Optimal damper mass of two damper types relative to engine order.

$F_{\mathrm{CG}}$ - and, therefore, the contact normal forces-acting on the damper decrease, following

$$
F_{\mathrm{CG}}=\frac{m R \Omega_{\mathrm{E}}^{2}}{E O^{2}}
$$

where $m$ denotes the damper mass and $R$ is the distance of the $\mathrm{CG}$ of the damper to the rotor axis. To compensate for the lower contact normal forces at higher engine orders, the damper mass has to be increased to get reasonable damping effects at higher engine orders.

Because of a certain contact height, pure rolling is excluded in the case of the wedge-shaped damper, even in the event of in-phase blade vibration $(E O=0)$. Thus, for small relative displacements caused by lower engine orders, the wedge-shaped damper is preferable. Nevertheless, the disadvantage of a possible partial lift-off by the wedge-shaped damper from the blade platforms at the damper's edges must be noted. This lift-off occurs, for example, at higher vibration amplitudes caused by large excitation forces and even at small vibration amplitudes if the contact height is too large or slight manufacturing tolerances prevent the damper from perfectly fitting between the blade platforms. (Compare Fig. 2.) Neglecting the platform and damper elasticity in the normal direction and assuming rigid structures would lead immediately to a gap between the damper and the platforms, resulting in a line contact at the damper edges. Considering normal elasticities, a certain rolling of the wedgeshaped damper is included, and a possible separation from the platforms can be detected by the vibration amplitudes in the normal $(\zeta-)$ direction and the initial contact normal forces in the static equilibrium position of the damper due to the centrifugal forces. In Figure 9, a typical distribution of the vibration amplitudes in the normal direction is given for all point contact elements of the discretized contact area. Here, $\xi^{*}=\xi / h_{\mathrm{C}}$ and $\eta^{*}=\eta / \ell_{\mathrm{C}}$ denote the normalized contact height and the normalized contact length, respectively. The equation $r=\left|\hat{w}_{u_{\zeta}} / \bar{w}_{u_{\zeta}}\right|$ indicates the ratio of the vibration amplitude $\hat{w}_{u_{\zeta}}$ of every single 


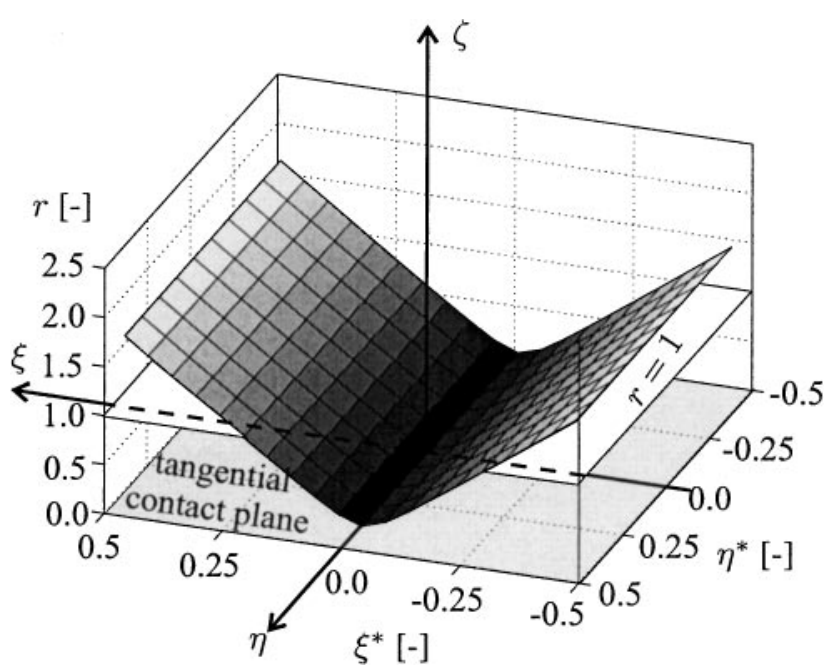

FIGURE 9

Possible partial lift-off of the wedge-shaped damper at the damper's edges.

point contact element in the normal direction, perpendicular to the contact plane, with respect to the static equilibrium position $\bar{w}_{u_{\zeta}}$ in the normal direction. The latter is determined by the normal contact stiffness and the normal contact forces caused by the centrifugal forces $F_{\mathrm{CG}}$. A value of $r>1$ indicates that separation occurs during one vibration cycle and occurs primarily at the damper edges, $\xi^{*}= \pm 0.5$.

In the case of the cylindrical damper, the effectiveness and the position between the platforms is less sensitive with regard to manufacturing tolerances. Even in the case of large vibration amplitudes, a cylindrical damper always fits into a well-defined position between the platforms because of its curved surfaces, leading to Hertzian line contacts. Some possible strategies to improve the design of cylindrical dampers in order to avoid the rolling condition and increase the damping effect are presented here.

\section{Improvement of the Damper Design}

One possible modification to minimize the rolling of a cylindrical damper in the case of small or even vanishing relative displacements is to break the symmetry of the blade-damperblade contact. So the same 16-blade assembly presented earlier was subjected to an engine order 1 excitation.

Figure 10 shows the optimization curves as presented in Figure 6 for a constant platform angle $\gamma_{\mathrm{L}}=35^{\circ}$ at the left contact and a varying platform angle $\gamma_{\mathrm{R}}=15^{\circ} \ldots 85^{\circ}$ at the right contact. Starting from the reference configuration of symmetrical platforms $\left(\gamma_{R}=\gamma_{L}\right)$, a decrease or increase of the right platform angle leads to a better damping effect if the respective optimal damper mass is chosen for each configuration. Because of contact kinematics, the relative displacements in the contact areas have changed, and the damping potential can be increased distinctly. Furthermore, it is evident that a variation in any system

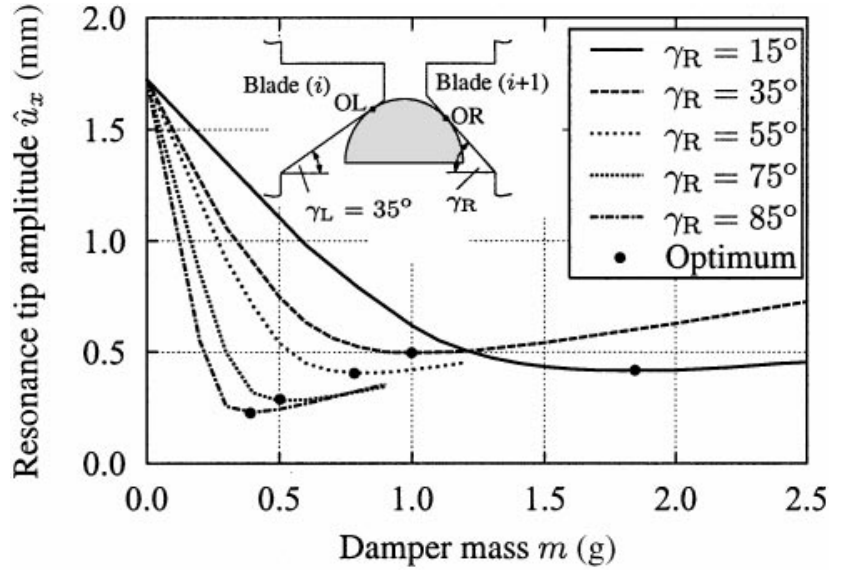

FIGURE 10

Variation in platform angle $\gamma_{R}$ to improve the damping effects.

parameter, such as the contact geometry, requires a reoptimization of the damper mass so as to take into consideration the changed contact conditions, in particular the different contact normal forces acting in the left and right contacts. This becomes obvious in the case of $\gamma_{\mathrm{R}}=15^{\circ}$, where a reoptimized damper mass leads to a reduction, and a constant mass of $m=1 \mathrm{~g}$ to an increase in the resonance amplitude in comparison with $\gamma_{\mathrm{R}}=35^{\circ}$. Because of the blade's symmetry, the same results are obtained if the left and right platform angle are exchanged.

The consequences of a change to asymmetrical platform angles can be studied in terms of the relative displacements and contact forces in $\xi$-direction, which is the predominant direction of the relative displacements in the first bending mode. In Figure 11, the real and imaginary parts of the normalized complex relative displacements $\hat{w}_{u_{\xi}}^{*}$ and the corresponding tangential contact forces $\hat{F}_{\mathrm{C} \xi}^{*}$ are given in the complex plane.

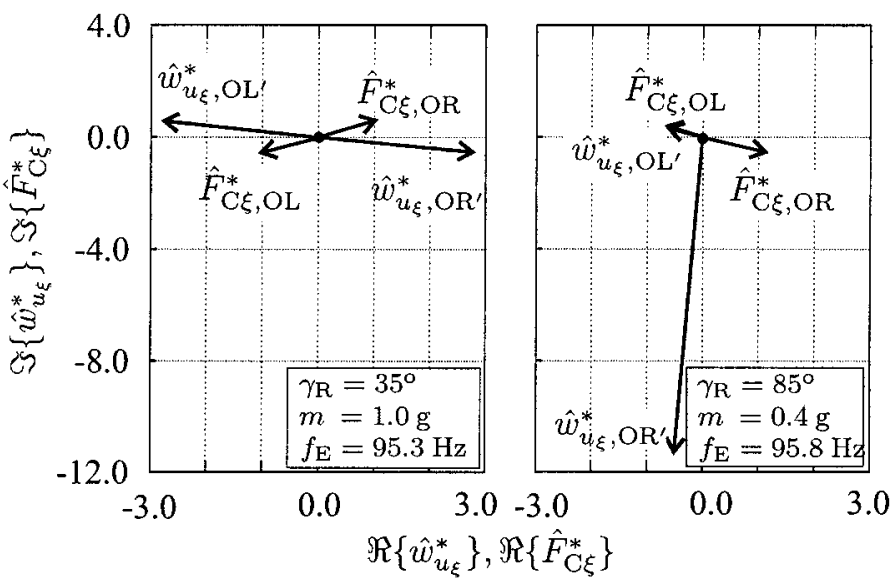

FIGURE 11

Complex normalized relative displacements and normalized contact forces in $\xi$-direction in the case of resonance for $\gamma_{\mathrm{R}}=35^{\circ}$ and $\gamma_{\mathrm{R}}=85^{\circ}$. 
The left plot indicates the symmetrical case $\gamma_{R}=\gamma_{L}=35^{\circ}$, the right plot the asymmetrical case with $\gamma_{R}=85^{\circ}$, while OL denotes the left and OR the right control point. The relative displacements and contact forces acting between the control points $\mathrm{OL}, \mathrm{OL}^{\prime}$, and $\mathrm{OR}, \mathrm{OR}^{\prime}$, respectively, are normalized by

$$
\hat{w}_{u_{\xi}}^{*}=\frac{\hat{w}_{u_{\xi}} c_{\mathrm{T}}}{\mu F_{\mathrm{N}}}, \quad \hat{F}_{\mathrm{C} \xi}=\frac{\hat{F}_{\mathrm{C} \xi}}{\mu F_{\mathrm{N}}}
$$

where $c_{\mathrm{T}}$ denotes the global tangential contact stiffness, $\mu$ the coefficient of friction, and $F_{\mathrm{N}}$ the total contact normal force; $F_{\mathrm{N}, \mathrm{OL}} \neq F_{\mathrm{N}, \mathrm{OR}}$ in the asymmetrical case.

In the symmetrical case, the relative displacements and the corresponding contact forces at both contacts show an observable phase difference, which is a necessary condition to cause energy-dissipating friction forces. The condition at the left and right contacts are equal, with a phase shift of $180^{\circ}$, which should be expected to meet the equilibrium conditions at the damper. In the asymmetrical case of $\gamma_{R}=85^{\circ}$ it becomes evident that the normalized relative displacement $\hat{w}_{u_{\xi}, \mathrm{OL}^{\prime}}^{*}$ at the left contact is smaller than 1 , indicating that sticking occurs. Therefore, the generated tangential contact forces $\hat{F}_{\mathrm{C} \xi \text {,OL }}^{*}$ are nearly in phase with the displacement and no damping is provided, yielding $\hat{F}_{\mathrm{C} \xi, \mathrm{OL}}^{*} \approx \hat{w}_{u \xi, \mathrm{OL}}^{*}$. Because of the asymmetrical platforms, the normal contact force acting at the right contact is much less than that at the left contact to satisfy the equilibrium equation of the damper under given centrifugal forces. This results in relative displacements at the right contact with $\hat{w}_{u_{\xi}, \mathrm{OR}^{\prime}}^{*}>1$; that is, the damper is sliding, and corresponding friction forces $\hat{F}_{\mathrm{C} \xi, \mathrm{OR}}^{*}$ are generated (Fig. 11). The force/displacement relationships are given by the amplitude-dependent complex contact stiffnesses $\hat{k}_{u_{\xi}, \text { OL }}$ and $\hat{k}_{u_{\xi}, \text { OR }}$ from the contact stiffness matrix $\hat{\mathbf{K}}_{\mathrm{C}}$ given in Equation (10).

(a)

(b)

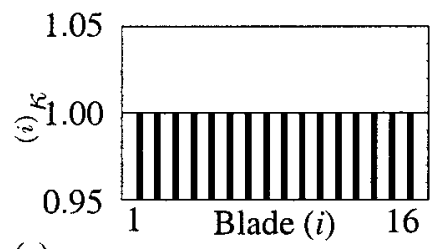

(c)
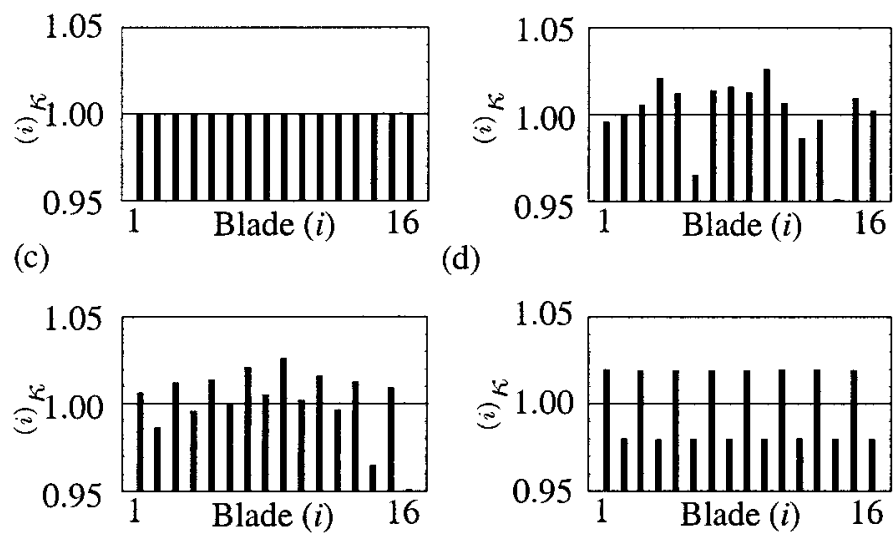

(d)

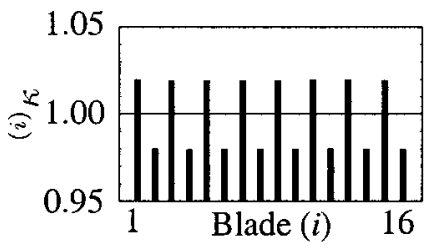

FIGURE 12

Mistuning factors ${ }^{(i)} \kappa$ : (a) tuned system; (b) statistical mistuning; (c) reordered configuration of (b); (d) systematical mistuning.

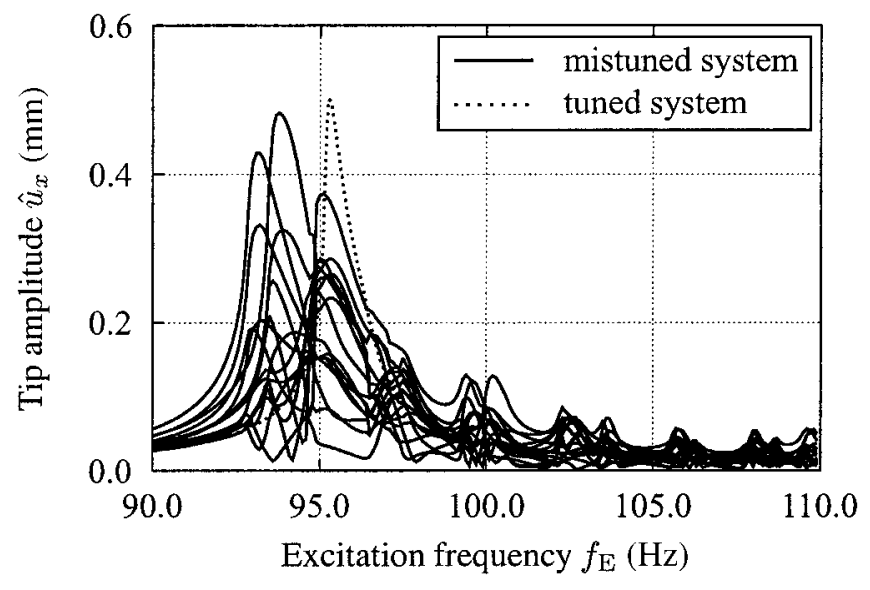

FIGURE 13

Statistical mistuning with $m=1 \mathrm{~g}$; random arrangement of the blades.

\section{MISTUNED SYSTEM}

As a result of manufacturing tolerances, real periodic systems like bladed disk assemblies always show some deviations and perturbations of the perfectly tuned cyclic system. There can be parameter variations concerning the dynamic properties of the blades as well as concerning the contact conditions. We studied frequency mistuning of the blades, assuming the same 16-blade reference model as used before, with symmetrical contact conditions $\gamma_{\mathrm{L}}=\gamma_{\mathrm{R}}=35^{\circ}$.

In the mistuned system we investigated, the natural frequencies ${ }^{(i)} f_{0 j}$ of the blades were assumed to follow a Gaussian distribution, with a standard deviation of $2 \%$ and average values matching those of the tuned system $\bar{f}_{0 j}$ for each mode $j$. The natural frequencies can be expressed as ${ }^{(i)} f_{0 j}={ }^{(i)} \kappa \bar{f}_{0 j}$, with the mistuning factor ${ }^{(i)} \kappa$ for each blade (i) (Fig. 12).

Starting from the distribution of the tuned system given in Figure 12a, a statistical mistuning of the blades' natural

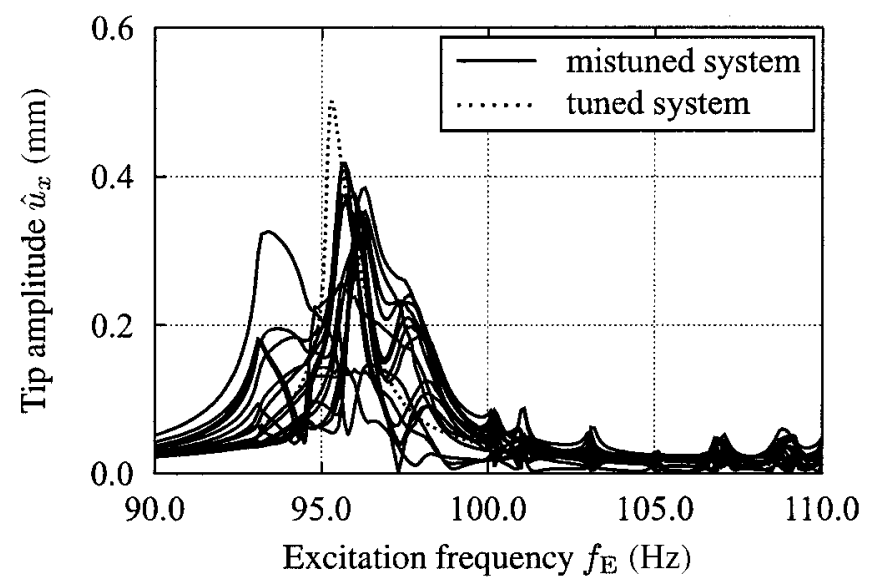

FIGURE 14

Statistical mistuning with $m=1 \mathrm{~g}$; reordered arrangement of the blades. 


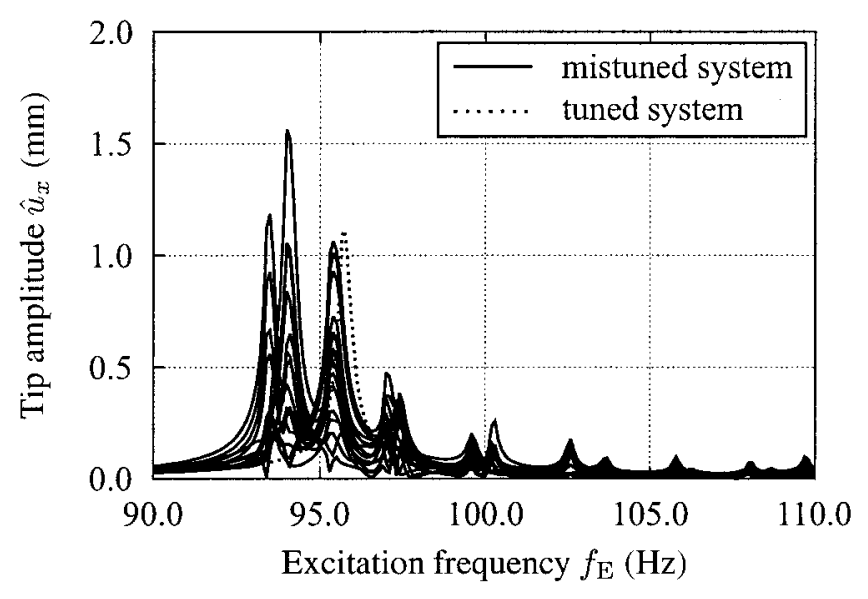

FIGURE 15

Statistical mistuning with $m=5 \mathrm{~g}$; random arrangement of the blades.

frequencies leads to the distribution given in Figure 12b with a random arrangement of the blades. In Figure $12 \mathrm{c}$ a rearrangement has been realized in order to avoid neighboring blades with similar natural frequencies and, therefore, small relative displacements. In Figure 12d a systematical, deliberate mistuning of ${ }^{(i)} f_{0 j}=(1 \pm 0.02) \bar{f}_{0 j}$ is introduced to obtain a cyclic, tuned system again with segments of two blades with alternating natural frequencies.

Figure 13 shows the FRF of all blades of the assembly in comparison with the tuned system for the case of a statistical mistuning and a random arrangement of the blades (case b). The damper mass is chosen to the optimal damper mass of the tuned system of $m=1 \mathrm{~g}$. It becomes evident that the largest resonance amplitude of a blade nearly equals the resonance amplitude of the tuned system. A closer view of the FRF of each blade provides the information that blade No. 14, whose natural frequencies

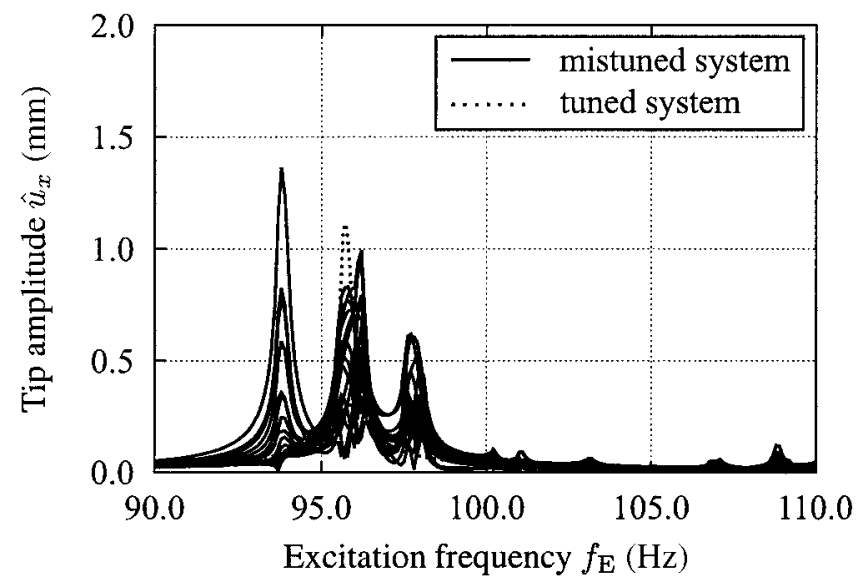

FIGURE 16

Statistical mistuning with $m=5 \mathrm{~g}$; reordered arrangement of the blades. vary greatly from those of its neighbors, experiences the lowest resonance amplitude. Reordering the blades (case c) gives the results depicted in Figure 14, with slightly lower resonance amplitudes. Again, the minimum amplitude can be detected for a blade with noticeable differences from the adjacent blades' natural frequencies, here blade No. 15. In both arrangements, the resonance amplitudes are slightly lower than in the case of a tuned system.

Further investigations of the mistuned system, choosing a damper mass differing from the optimal mass of the tuned system, showed that, according to the tuned system, the maximum resonance amplitude increases. Furthermore, several blades of the assembly experience a higher amplitude than that of a tuned system, as shown in Figure 15 for a damper mass of $m=5 \mathrm{~g}$. Again, it can be shown that in the case of a reordered arrangement of the blades, a slight amplitude reduction can be achieved in comparison with Figure 15, which nevertheless exceeds the resonance amplitude of the corresponding tuned system with $m=5 \mathrm{~g}$ (Fig. 16).

Furthermore, an amplitude reduction with respect to the tuned system is possible if the arrangement is detuned intentionally, for example, with alternating natural frequencies (case d), which is achievable by deliberate slight variations of the tuned blades' geometry or mass distribution. The results for $m=1 \mathrm{~g}$ are given in Figure 17. As mentioned earlier, the system shows cyclic symmetry with segments consisting of two blades. The vibration amplitudes of blades $1,3,5$, etc. are equal to those of blades 2, 4, 6, etc. According to the two-blade segment, two resonance frequencies occur. Even in the case of the first vibration mode, where the phase difference between neighboring blades is small and equals $\Delta \varphi=2 \pi E O / n$ of the tuned system, we achieved an amplitude reduction of about $38 \%$ (blade No. 1) and $15 \%$ (blade No. 2), respectively, in comparison with the tuned system.

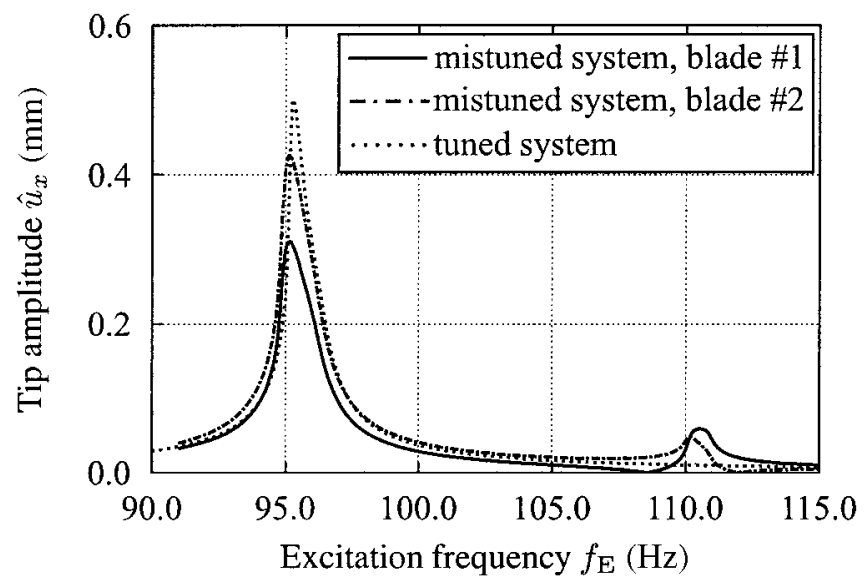

FIGURE 17

Amplitude reduction caused by systematic mistuning, with $m=1 \mathrm{~g}$. 


\section{CONCLUSIONS}

This article reports a computation method that has been developed to analyze tuned and mistuned bladed disk assemblies with cylindrical and wedge-shaped dampers. We applied a spatial contact model consisting of a number of discrete threedimensional point contact elements to study spatial blade vibrations. The analysis of a tuned system consisting of 16 identical blades showed the effectiveness of the different damper types for varying engine orders. It could be shown that the cylindrical damper tends to roll along the blade platforms for small relative displacements between neighboring blades caused by low engine orders and, thus, the damping potential decreases rapidly. While the wedge-shaped damper provides better damping effects and should be preferred in this case, this damper type tends toward a partial separation at its edges.

To improve the damper design and to avoid the damper's rolling, the influence of asymmetrical blade platforms was investigated; it showed that avoidance of symmetry increases the damping effect. Statistical and systematic mistuning of the blade assembly was analyzed, and it became evident that the optimal damper mass of the corresponding tuned system leads to similar vibration amplitudes in the mistuned system; on the other hand, a nonoptimal damper mass leads to higher vibration amplitudes than in the corresponding tuned system. Additionally, an intentional mistuning with alternating natural frequencies showed better damping potential by the damper than did the tuned system.

\section{REFERENCES}

Cha, D., and Sinha, A. 2000. Statistics of responses of a mistuned and frictionally damped bladed disk assembly subjected to white noise and narrow band excitation. ASME Paper 2000-GT-542. International Gas Turbine and Aeroengine Congress and Exhibit., Munich, Germany: ASME.

Chen, S., and Sinha, A. 1989. Probabilistic method to compute the optimal slip load for a mistuned bladed disk assembly with friction dampers. ASME Paper DE-18.3.

Csaba, G. 1999. Modelling of a microslip friction damper subjected to translation and rotation. ASME Paper 99-GT-149. International Gas Turbine and Aeroengine Congress and Exhibit., Indianapolis, IN.

Ewins, D. J. 1968. The effects of detuning upon the forced vibrations of bladed disk assemblies. Journal of Sound and Vibration 9:65-79.

Jareland, M. H., and Csaba, G. 2000. Friction damper mistuning of a bladed disk and optimization with respect to wear. ASME Paper
2000-GT-363. International Gas Turbine and Aeroengine Congress and Exhibit., Munich, Germany.

Jareland, M. H. 2001. A parametric study of a cottage roof damper and comparison with experimental results. ASME Paper 2001-GT-0275. International Gas Turbine and Aeroengine Congress and Exhibit., New Orleans, LA.

Jareland, M. H. 2001. Experimental investigation of a platform damper with curved contact areas. ASME Paper DETC2001/VIB-21391. Design Engineering Technical Conference, Pittsburgh, PA.

Judge, J., and Pierre, C. 2000. Experimental investigation of mode localization and forced response magnification for a mistuned bladed disk. ASME Paper 2000-GT-358. International Gas Turbine and Aeroengine Congress and Exhibit., Munich, Germany.

Menq, C. H., Griffin, J. H., and Bielak, J. 1986. The influence of microslip on vibratory response. II: A comparison with experimental results. Journal of Sound and Vibration 107(2):295-307.

Mignolet, M. P., Hu, W., and Jadic, I. 1998. On the forced response of harmonically and partially mistuned bladed disks. I and II. Proceedings of the 7th International Symposium on Transport Phenomena and Dynamics of Rotating Machinery, Vol. B. Honululu, Hawaii.

Panning, L., Sextro, W., and Popp, K. 2000. Optimization of interblade friction damper design. ASME Paper 2000-GT-541. International Gas Turbine and Aeroengine Congress and Exhibit., Munich, Germany.

Pfeiffer, F., and Hajek, M. 1992. Sick-slip motion of turbine blade dampers. Philosophical Transactions of the Royal Society of London, Series A. 338:1651.

Sanliturk, K. Y., Ewins, D. J., and Stanbridge, A. B. 1999. Underplatform dampers for turbine blades: theoretical modelling, analysis and comparison with experimental data. ASME Paper 99-GT-335. International Gas Turbine and Aeroengine Congress and Exhibit., Indianapolis, IN.

Sextro, W., Popp, K., and Wolter, I. 1997. Improved reliability of bladed disks due to friction dampers. ASME Paper 97-GT-189. International Gas Turbine and Aeroengine Congress and Exhibit., Orlando, FL.

Sextro, W., Popp, K., and Wolter, I. 1998. Three-dimensional vibration of bladed disk assemblies with friction dampers. In Proceedings of ISROMAC-7, the 7th International Symposium on Transport Phenomena and Dynamics of Rotating Machinery, Vol. B. Honolulu, Hawaii, 621-630.

Sextro, W. 1999. Forced vibration of elastic structures with friction contacts. ASME-DETC. No. DETC99/VIB-8180.

Yang, B. D., and Menq, C. H. 1997. Characterization of contact kinematics and application to the design of wedge dampers in turbomachinery blading, I and II. ASME Paper 97-GT-19/20. International Gas Turbine and Aeroengine Congress and Exhibit., Orlando, FL. 

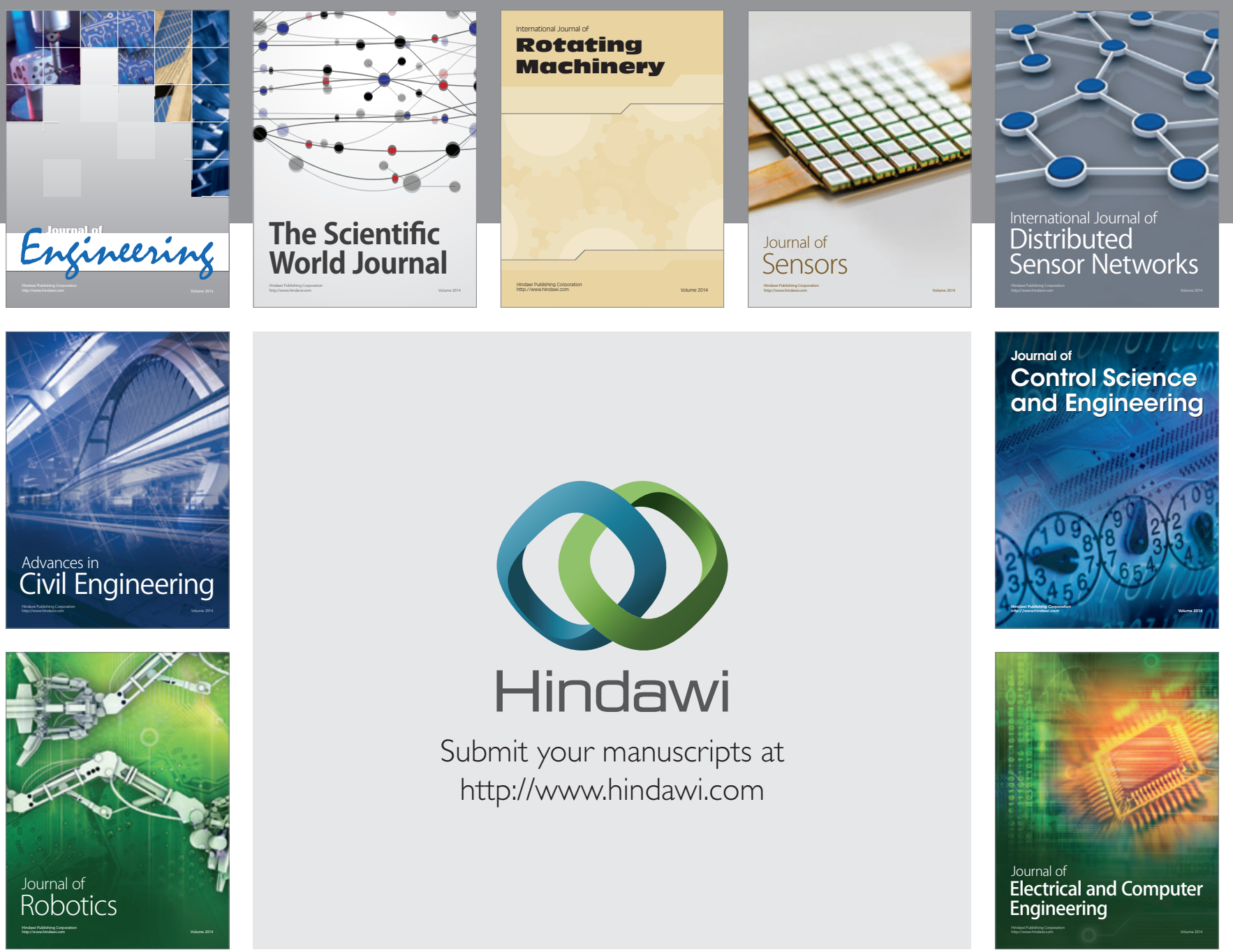

Submit your manuscripts at

http://www.hindawi.com
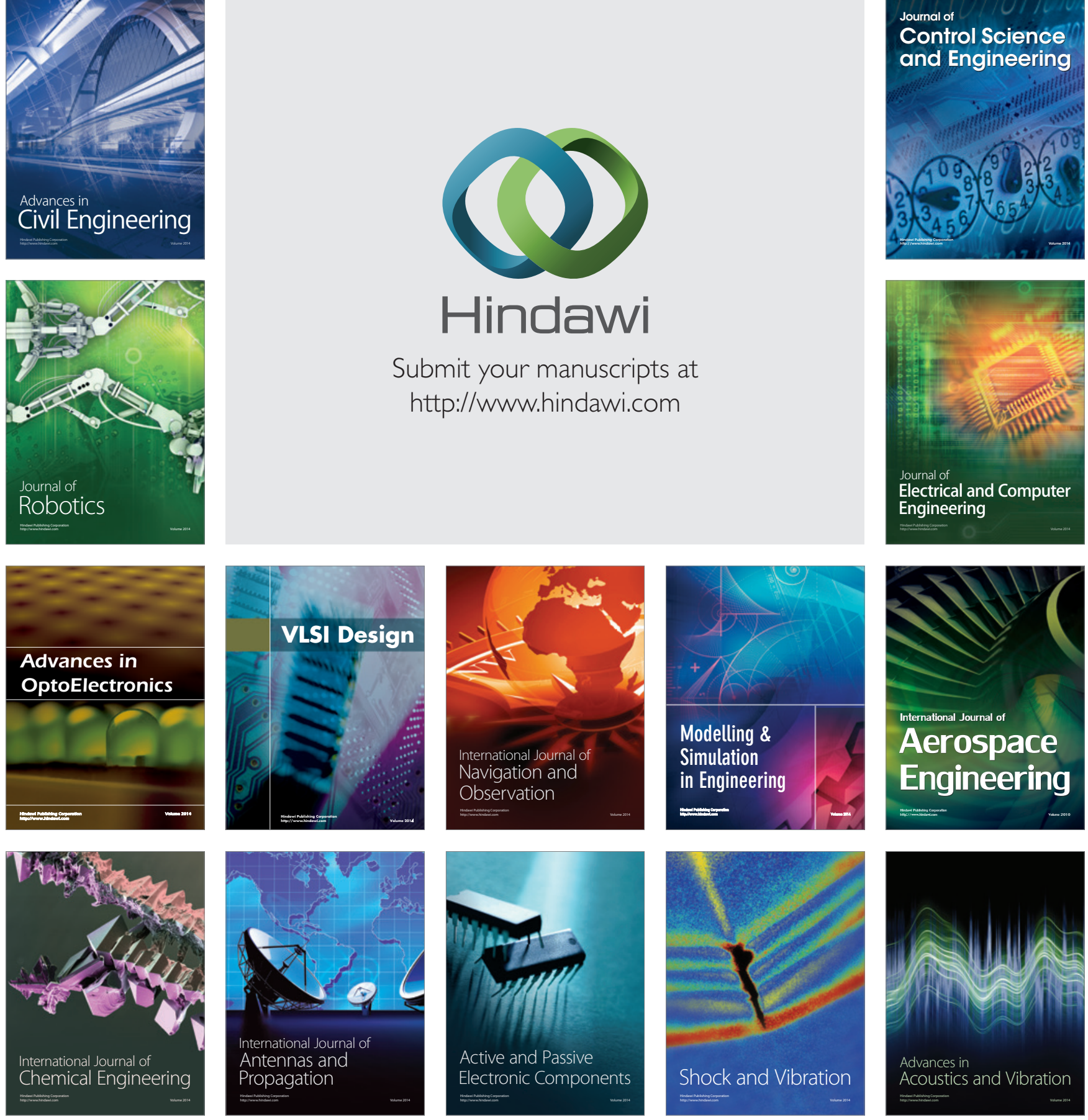